\title{
An analysis of dissipation functions in swarming systems
}

\author{
Derek J. Bennet* James D. Biggs * Colin R. McInnes* \\ Malcolm Macdonald* \\ * Advanced Space Concepts Laboratory, Department of Mechanical \\ Engineering, University of Strathclyde, Glasgow. \\ (e-mail: derek.bennet@strath.ac.uk; james.biggs@strath.ac.uk).
}

\begin{abstract}
Swarms of multiple, autonomous mobile agents have been shown to have advantages over single agent systems such as scalability, robustness and flexibility. This papers considers swarm pattern control using a generic artificial potential field and a range of dissipation control terms. An investigation of a number of dissipation terms to induce different swarm behaviours is undertaken. In addition, a novel dissipation control term is introduced based on time-delay feedback control. It is shown that a delayed dissipation term can induce vortex formations without knowledge of relative velocities. Finally, a stability analysis is undertaken that verifies swarm behaviour in a subset of these cases.
\end{abstract}

Keywords: swarming, artificial potential field, dissipation control, time-delay feedback control.

\section{INTRODUCTION}

In nature the term agent applies to swarms of social entities, such as insects, that self-organise through local communications and interaction with the environment. Instead of a centralised architecture, the agents in the swarm act in a distributed way, having no a priori knowledge of the pattern they will eventually relax into.

Beni has introduced the term swarm intelligence to describe a system that lacks any form of hierarchical control structure, where through microscopic interactions, macroscopic behaviour occurs (see Beni and Wang (1989)). The modelling of swarm intelligence has been researched extensively since the late 1980s, and as such there exists a wide array of approaches such as; cellular automata (Gardner (1970)), Reynolds flocking birds model (Reynolds (1987)), ant colony optimisation (Dorigo (1992)) and particle swarm optimisation (Kennedy and Eberhart (1995)).

One of the major research fields exploiting swarming is swarm robotics. Potential swarm robotics applications include; inspection of complicated engineering structures such as turbines with small miniature robots (Correll and Martinoli (2007)), scientific data gathering in formations of unmanned aerial vehicles (see McInnes (2003); Kingston et al. (2003); Frew et al. (2008); Quigley et al. (2005); Ollero and Merino (2004); Bennet and McInnes (2009b)) and for interferometric/sparse aperture telescopes in spacecraft formation-flying (see Carpenter et al. (2004); Wallner (2006)).

The control of multiple robots can be split broadly into either centralised or decentralised control. As opposed to the traditional AI approach, Brooks introduced his behaviouralbased approach that is directly related to the swarm intelligence paradigm (Brooks (1986)). Instead of requiring an internal model of the environment and some form of cognitive ability, the behaviour-based approach is based on a collection of basic behaviours, which either act independently or dependently, to produce an emergent behaviour. Brooks implemented this idea in his bottom-up subsumption based architecture, that consists of a set of layered independent behaviours, with higher level behaviours subsuming lower level ones. Although, this approach has been shown to produce interesting emergent behaviours, the systems lacks formality and is therefore difficult to validate, an essential requirement for safety or mission critical applications.

This paper considers the use of a fusion behaviour-based approach known as the artificial potential field (APF) Reif and Wang (1999); Gazi and Passino (2002); Chang et al. (2003); Ogren et al. (2004); D'Orsogna et al. (2006b); Kim et al. (2006); Bennet and McInnes (2009a); Khatib (1986) alongside a number of dissipation controls. Specifically, we consider a swarm of homogeneous, autonomous agents $(1 \leq i \leq N)$ that are interacting through an APF $U\left(\mathbf{x}_{i}\right)$, explicitly where:

$$
U\left(\mathbf{x}_{i}\right)=\sum_{j, j \neq i}\left[C_{r} \exp ^{-\frac{\left|\mathbf{x}_{i j}\right|}{L_{r}}}-C_{a} \exp ^{-\frac{\left|\mathbf{x}_{i j}\right|}{L_{a}}}\right]+\frac{\alpha}{2}\left|\mathbf{x}_{i}\right|^{2}
$$

where, $\mathbf{x}_{i}$ is the position vector of agent $i$ and $\mathbf{x}_{i j}$ is the relative position vector of agents $i$ and $j, C_{a}, C_{r}$ and $L_{a}, L_{r}$ represent the amplitude and range of the attractive and repulsive potential respectively, and $\alpha$ represents the amplitude of a quadratic potential. This potential function comprises the Morse potential function and a quadratic potential term $\frac{\alpha}{2}\left|\mathbf{x}_{i}\right|^{2}$. These components of the pair-wise potential are illustrated in Fig. 1 This Morse potential, as shown in Fig. 1 is used to provide longrange attraction and weak short-range repulsion (collision free motion) for the swarm of agents (D'Orsogna et al. (2006a)). The quadratic potential, shown in Fig. 1 (ii), ensures that formations are driven to the origin. In this paper we investigate swarm behaviour induced by the following equations of motion: 


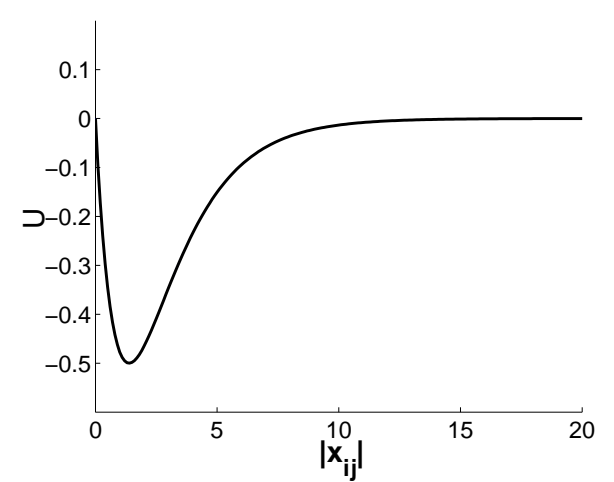

(i)

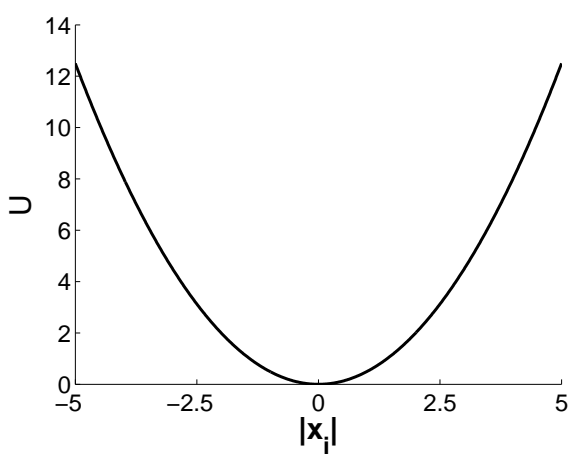

(ii)

Fig. 1. Artificial potential field (i) Morse potential (ii) quadratic

$$
m \dot{\mathbf{v}}_{i}=-\nabla_{i} U\left(\mathbf{x}_{i}\right)+\mathbf{u}(t)
$$

where $U\left(\mathbf{x}_{i}\right)$ is the potential function defined in (1) and $\mathbf{u}(t)$ is the dissipation control term. In particular, we consider the following control terms and investigate the induced swarm behaviour.

(1) A simple velocity dependent dissipation term

$$
\mathbf{u}(t)=-\sigma \mathbf{v}_{i}
$$

where, $\mathbf{v}_{i}$ is the velocity vector of agent $i$ and $\sigma$ is a constant controlling the amplitude of the dissipation.

(2) A dissipation term that aligns the agent's velocities

$$
\mathbf{u}(t)=\sum_{i \neq j} C_{o}\left(\mathbf{v}_{i j} \cdot \hat{\mathbf{x}}_{i j}\right) \exp ^{-\left|\mathbf{x}_{i j}\right| / L_{o}} \hat{\mathbf{x}}_{i j}
$$

where, $\mathbf{v}_{i j}$ is the relative velocity vector of agents $i$ and $j$, $C_{o}$ and $L_{o}$ represent the strength and range of the orientation function and $(\hat{\bullet})$ denotes a unit vector.

(3) A dissipation term that tracks a pre-specified signal

$$
\mathbf{u}(t)=\left[\begin{array}{l}
-\sigma v_{x}-\nabla_{i} U\left(x_{i j}\right)-\alpha\left(x_{i}-\xi \sin (t)\right) \\
-\sigma v_{y}-\nabla_{i} U\left(y_{i j}\right)-\alpha\left(y_{i}-\zeta \cos (t)\right)
\end{array}\right]
$$

where, $\mathbf{x}=\left[x_{i j}, y_{i j}\right]^{T}$ and $\xi, \zeta$ control the shape of the desired trajectory.

(4) A time-delayed dissipation term

$$
\mathbf{u}(t)=-K\left(v_{i}(t)-v_{i}(t-\tau)\right)
$$

where $K$ is the magnitude of the dissipation term and $\tau$ is the delay term.
Note that the dissipation terms described in (3) and (4) can be found in Bennet and McInnes (2009b); Mabrouk and McInnes (2008). However, using Time-delay feedback control (Timedelayed auto synchronization) as a method to autonomously control swarms of interacting particles is new. In this paper we simulate these cases to illustrate varying induced swarm patterns. In addition, a stability analysis of the first two cases is undertaken. The stability analysis of the second two cases will by undertaken in future research.

The time-delayed dissipation term is a novel method for inducing swarm behavior. It is based on the method of TimeDelay-Autosynchronization (T-DAS) also refered to as timedelay feedback control. This is conventionally used as a method stabilize unstable periodic orbits (UPOs) embedded in chaotic attractors in systems of ordinary differential equations. T-DAS was first proposed by Pyragas (1992) as a method that requires no exact knowledge of the form of the periodic orbit or even the form of the original dynamics. This method has been successful in a number of applications and has recently found its way into the aerospace literature Biggs and McInnes (2009).In this paper we propose to apply a T-DAS type methodology with the aim of inducing periodic motion in swarms.

\section{SWARM SIMULATIONS}

The following simulations were carried out:

\subsection{Case (i)}

Consider the case when the term is velocity dependent as shown in Eq. 3;

A swarm of 50 agents are given random initial conditions, as shown in Fig. 2 (i). As time evolves the swarm dissipates energy and relaxes into a local minimum energy configuration, as shown in Fig. 2 (ii.)

\subsection{Case (ii)}

Equation 4 shows the dissipation function that leads to the constrained minimisation of the total effective energy and conservation of the total angular momentum of the swarm (McInnes (2007)).

Figure 3 shows the results for a swarm of 30 agents that are given random initial conditions.

From the results shown in Fig. 3 it can be seen that the swarm will relax into an equally spaced rotating vortex pattern, through the minimisation of the total energy and conservation of angular momentum, as will be explained later is Section 3.

\subsection{Case (iii)}

Equation 5 shows the case when the dissipative term allows the swarm centre-of-mass to track a desired trajectory.

Two cases are considered for varying values of $\xi$ and $\zeta$, as summarised in Table 1. The results are shown in Fig. 4 and 5 for 


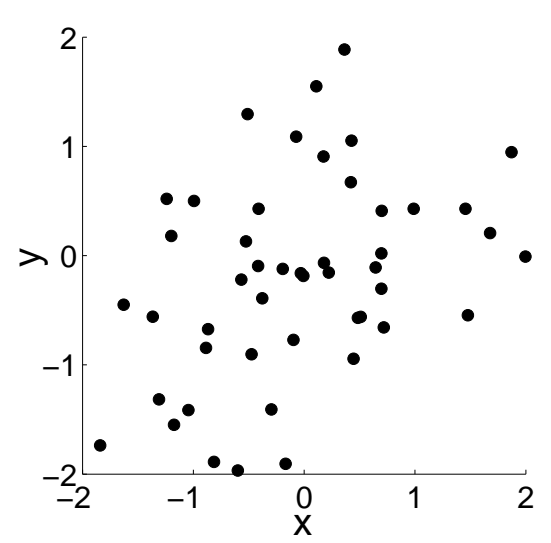

(i)

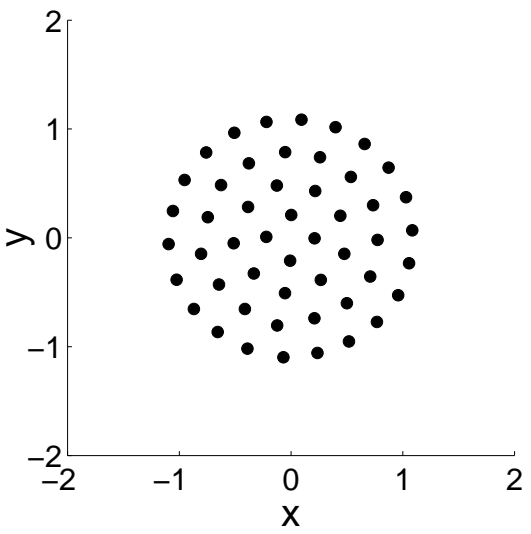

(ii)

Fig. 2. Pair-wise pattern formation (i) random initial conditions (ii) equally spaced cluster $\left(C_{a}=2, L_{a}=2, C_{r}=2, L_{r}=1\right.$, $m=1, \sigma=1$ and $\alpha=2$ )

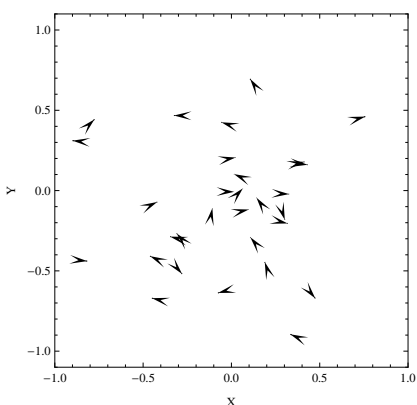

(i)

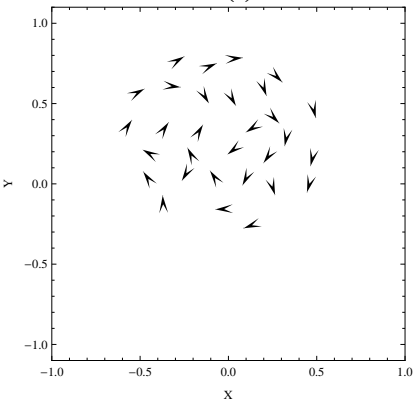

(iii)

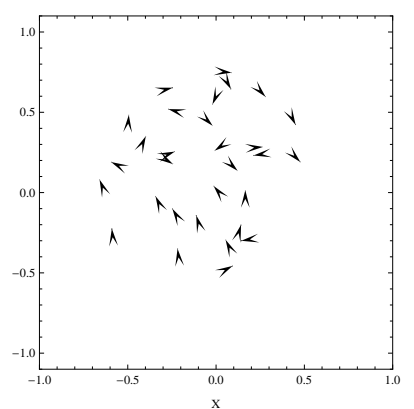

(ii)

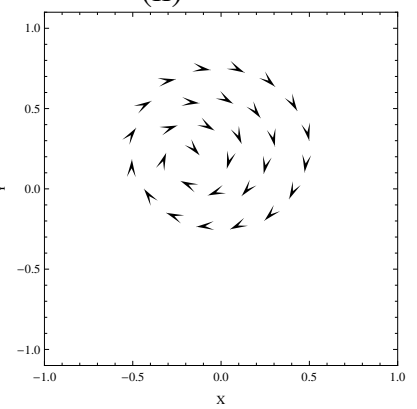

(iv)
Fig. 3. Swarm orientation function (i) random initial conditions (ii) time $=5 \mathrm{~s}$ (iii) time $=10 \mathrm{~s}$ (iv) time $=40 \mathrm{~s}$ the formation at different times with random initial conditions. From these results it can be seen that the swarm center-of-mass can track the path defined by the parameters $\xi$ and $\zeta$ whilst maintaining a swarm cluster formation. If $\xi$ and $\zeta$ are equal a circular path will be obtained for the formation, as shown in Fig. 4. However, if $\xi, \zeta$ are different an elliptical path can be generated, as shown in Fig. 5.

Table 1. Swarm centre-of-mass parameters

\begin{tabular}{cccccccccc}
\hline \hline path & $C_{a}$ & $L_{a}$ & $C_{r}$ & $L_{r}$ & $m$ & $\sigma$ & $\alpha$ & $\xi$ & $\zeta$ \\
\hline circular & 2 & 2 & 2 & 1 & 1 & 2 & 10 & 2 & 2 \\
ellipse & 2 & 2 & 2 & 1 & 1 & 2 & 10 & 4 & 2
\end{tabular}

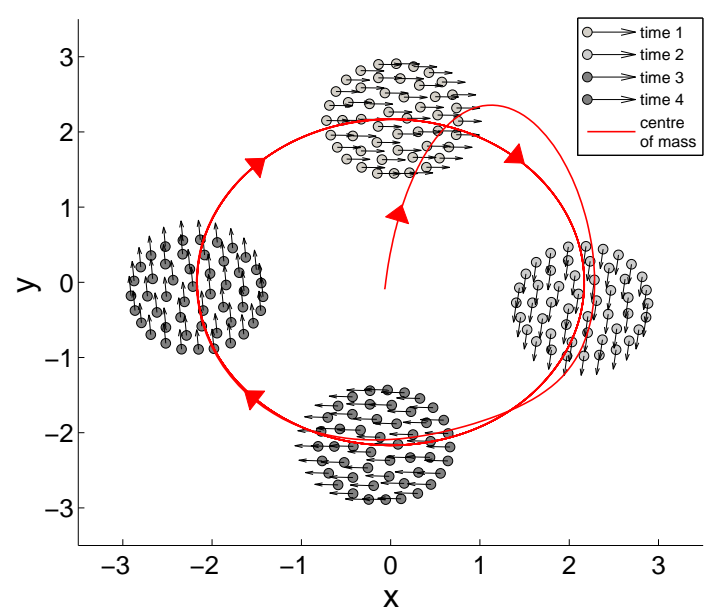

Fig. 4. Swarm centre-of-mass circular path

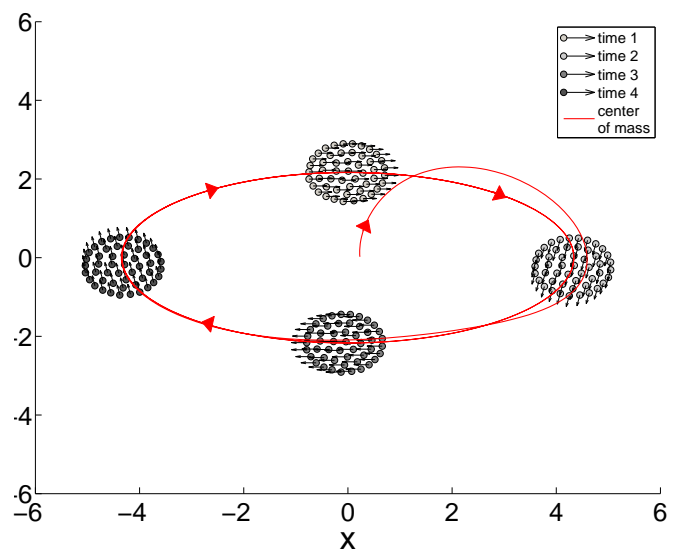

Fig. 5. Swarm centre-of-mass elliptical path

\subsection{Case (iv)}

In this case we will consider the dissipation term $\Upsilon_{i}$ defined by equation (6), as shown in Fig. 6.

Intuitively this dissipation term will force each particle to trace its delayed path. Note that if this term forces each particle of the swarm into a periodic oscillation of period that is equal to $\tau$ then the dissipation term will be zero. 


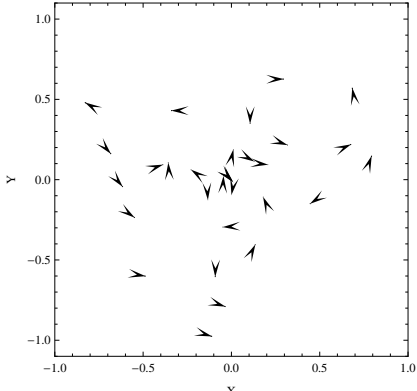

(i)

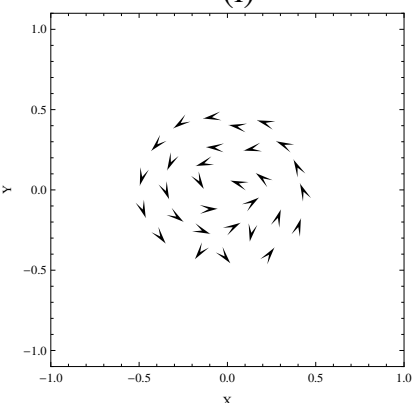

(iii)

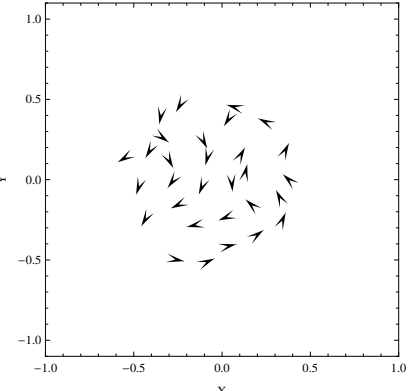

(ii)

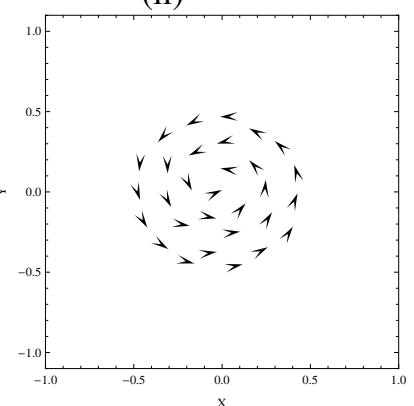

(iv)
Fig. 6. Swarm time-delay system (i) random initial conditions (ii) time $=10 \mathrm{~s}$ (iii) time $=20 \mathrm{~s}$ (iv) time $=40 \mathrm{~s}$

From the simulation it can be seen that the time-delay dissipation term induces vortex type behavior in the swarm, similar to that shown for Case (ii). However, the time-delayed term would have an advantage in real-world applications as it only requires the agent to know its current state along with its state one period in the past in-order to induce the desired behavior. The dissipation term in Case (ii), by contrast, requires all the relative velocities between each agent to be known.

\section{STABILITY}

A significant advantage of modelling the swarm through the APF method is that verifiable swarming can be developed, replacing traditional heuristic methods with a more rigorous analytical approach. This section discusses the stability of case (i) and (ii), proving the emergence of the formations analytically. The stability of case (iii) and case (iv) can be considered as part of future work.

\subsection{Case (i) stability}

Taking the dot product of the velocity vector with Eq. 2 and summing over all the agents results in;

$$
\sum_{i} m \mathbf{v}_{i} \cdot \dot{\mathbf{v}}_{i}=-\sum_{i} \nabla_{i} U\left(\mathbf{x}_{i}\right) \cdot \mathbf{v}_{i}-\sum_{i} \sigma \mathbf{v}_{i}^{2}
$$

Thus, the rate of change of total effective energy, $E$, of the system is continually decreasing, as shown in Eq. 8, until the system reaches an equilibrium state;

$$
\frac{d E}{d t}=-\sigma \sum_{i} \mathbf{v}_{i}^{2} \leq 0
$$

where, $E=\frac{1}{2} \sum_{i} m \mathbf{v}_{i}^{2}+\sum_{i} U\left(\mathbf{x}_{i}\right)$.
Also, taking the cross product of the position vector with Eq. 2 and summing over all the agents results in;

$$
\sum_{i} m \mathbf{x}_{i} \times \dot{\mathbf{v}}_{i}=-\sum_{i} \mathbf{x}_{i} \times \nabla_{i} U\left(\mathbf{x}_{i}\right)-\sum_{i} \sigma \mathbf{x}_{i} \times \mathbf{v}_{i}
$$

Defining the angular momentum, $\mathbf{H}=\sum_{i} \mathbf{x}_{i} \times \mathbf{v}_{i}$, it can be shown that the rate of change of angular momentum will also be continually decreasing until the system reaches an equilibrium state as shown in Eq. 10;

$$
\frac{d \mathbf{H}}{d t}=-\left(\frac{\sigma}{m}\right) \mathbf{H} \leq 0
$$

since, $\sum_{i} \mathbf{x}_{i} \times \nabla_{i} U\left(\mathbf{x}_{i}\right)=0$ due to internal symmetry in the swarm.

Finally, consider the position vector of the center-of-mass, shown in Eq. 11;

$$
\mathbf{R}_{c}=\frac{\sum_{i} m \mathbf{x}_{i}}{\sum_{i} m}
$$

By summing over all the agents in Eq. 2, it can be shown that the swarm can be controlled through its center-of-mass, given in Eq. 12;

$$
M \ddot{\mathbf{R}}_{c}+\sigma \dot{\mathbf{R}}_{c}+\alpha \mathbf{R}_{c}=0
$$

where, $M=\sum_{i} m$ and noting that $\sum_{i} \nabla_{i} U\left(\mathbf{x}_{i j}\right)=0$ due to internal symmetry in the swarm.

The eigenvalues for Eq. 12 are $\lambda_{1,2}=\frac{1}{2 M}\left[-\sigma \pm \sqrt{\left(\sigma^{2}-4 \alpha M\right)}\right]$. For $\sigma>0$ and $\alpha>0$ the eigenvalues are always either negative real or complex with negative real part so that the centre-ofmass of the swarm can be considered stable. For the results shown in Fig. 2 the eigenvalues of the system are $\lambda_{1,2}=-1 \pm \mathrm{i}$ so that the centre-of-mass should follow a stable spiral to the origin, as confirmed in Fig. 7 (i). It can also be seen in Fig. 7 (ii) that the rate of change of angular momentum is continually decreasing until equilibrium is reached, as stated in Eq. 10.

\subsection{Case (ii) stability}

In a similar procedure to that shown previously, taking the dot product of the velocity vector with Eq. 2 and summing over all agent states results in;

$$
\frac{d E}{d t}=-\sum_{i} \mathbf{v}_{i} \mathbf{u}(t) \leq 0
$$

where $\mathbf{u}(t)$ is defined by Eq. 2. See the paper Mabrouk and McInnes (2008) for a detailed proof. Also, taking the cross product of the position vector with Eq. 2 and summing over all agent states it can be shown that; 


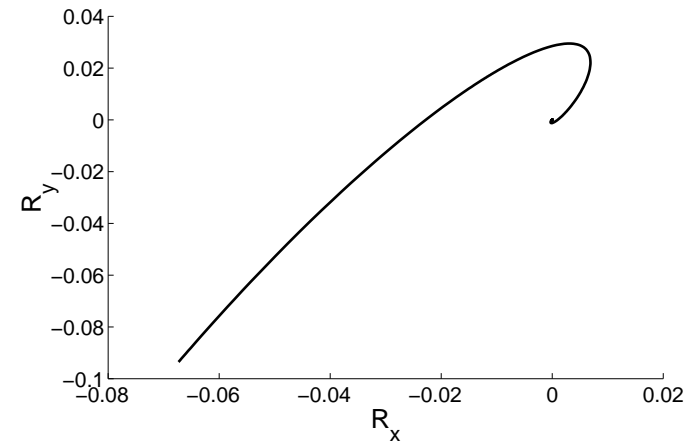

(i)

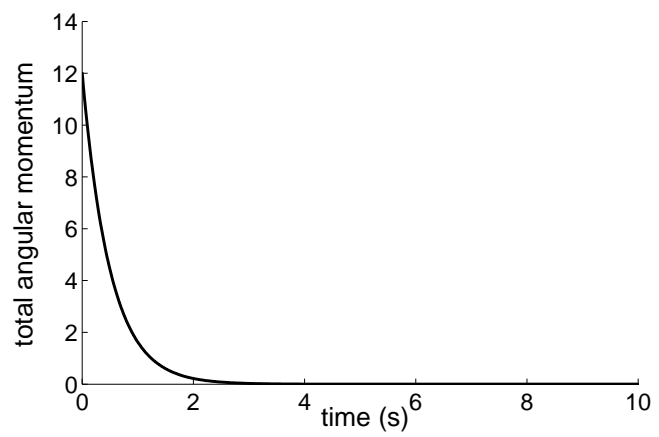

(ii)

Fig. 7. Swarm static formation (i) motion of centre-of-mass to origin $\left(\mathbf{R}_{c}=\mathbf{R}_{x} \mathbf{i}+\mathbf{R}_{y} \mathbf{j}\right)$ (ii) angular momentum

$$
\frac{d \mathbf{H}}{d t}=0
$$

The verification and stability analysis of the time-delay induced swarm will be the subject of future research. This stability analysis will combine the methods described in this paper used to address the stability of different dissipation terms alongside the stability analysis of time-delay systems. The implications on the stability analysis of the introduction of a time-delayed term implies the use of tools for stability of infinite dimensional systems.

\section{CONCLUSION}

This paper has considered swarm pattern control through the use of a generic artificial potential field and a range of dissipation control terms. It was shown that depending on the form of the dissipation function different swarm patterns can be induced. In addition, a novel dissipation control term was introduced based on time-delay feedback control which induces vortex formation. The stability of the swarm systems was investigated for a subset of the cases considered, showing that swarm verification can be achieved analytically. Future work will consider other dissipation terms as well as verifying the stability of the time-delay system.

\section{REFERENCES}

Beni, G. and Wang, J. (1989). Swarm intelligence in cellular robotic systems. In Proceedings NATO Advanced Workshop on Robots and Biological Systems. Tuscany, Italy.

Bennet, D. and McInnes, C. (2009a). Distributed control of multi-robot systems using bifurcating potential fields.
In Journal of Robotics and Autonomous Systems. Elsevier Science Ltd., ISSN: 0921-8890. IF-1.214, HL-5.8.

Bennet, D. and McInnes, C. (2009b). Verifiable control of a swarm of unmanned aerial vehicles. Proceedings of the Institution of Mechanical Engineers, Part G, Journal of Aerospace, 223(7), 939-953.

Biggs, J.D. and McInnes, C. (2009). Time-delayed feedback control in astrodynamics. Journal of guidance, dynamics and control., 31(6), 1804-1811.

Brooks, R. (1986). The robust layered control system for a mobile robot. IEEE Transactions of Robotics and Automation, 2(1), 14-23.

Carpenter, K., Schrijver, C., Allen, R., Brown, A., Chenette, D., Danchi, W., Karovska, M., Kilston, S., Lyon, R., Marzouk, J., Mazzuca, L., Moe, R., Walter, F., and Murphy, N. (2004). The stellar imager (SI): a revolutionary large-baseline imaging interferometer at the Sun-Earth $\mathrm{L}_{2}$ point. New Frontiers in Stellar Interferometry, 5491(1), 243-254.

Chang, D., Shadden, S., Marsden, J., and Olfati-Saber, R. (2003). Collision avoidance for multiple agent systems. In Proceedings of 42nd IEEE Conference on Decision and Control, volume 1, 539-543. Maui, Hawaii, USA.

Correll, N. and Martinoli, A. (2007). A challenging application in swarm robotics: The autonomous inspection of complex engineered structures. Bulletin of the Swiss Society for Automatic Control, 46, 15-19.

Dorigo, M. (1992). Optimization, Learning and Natural Algorithms. Ph.D. thesis, Politecnico di Milano.

D’Orsogna, M., Chuang, Y., Bertozzi, A., and Chayes, S. (2006a). Self-propelled particles with soft-core interactions: patterns, stability and collapse. Physical Review Letters, 96(10), 104302.

D’Orsogna, M., Y.L., C., A.L., B., and S., C. (2006b). The road to catastrophe: stability and collapse in 2D driven particle systems. In Hawaii International Conference on Statistics, Mathematics and Related Fields. Honolulu, Hawaii, USA.

Frew, E., Lawrence, D., and Morris, S. (2008). Coordinated standoff tracking of moving targets using lyapunov guidance vector fields. Journal of Guidance, Control and Dynamics, 31(2), 290-306.

Gardner, M. (1970). The fantastic combinations of John Conway's new solitaire game "life". Scientific America, 223, $120-123$.

Gazi, V. and Passino, K. (2002). A class of attraction/repulsion functions for stable swarm aggregations. In Proceedings of the 41st IEEE Conference on Decision and Control, volume 3, 2842-2847. Las Vegas, Nevada, USA.

Kennedy, J. and Eberhart, R. (1995). Particle swarm optimization. In Proceedings of the IEEE International Conference on Neural Networks, 1942-1948. Piscataway, NJ, USA.

Khatib, O. (1986). Real-time obstacle avoidance for manipulators and mobile robots. The International Journal of Robotics Research, 5(1), 90-98.

Kim, D., Wang, H., and Shin, S. (2006). Decentralized control of autonomous swarm systems using artificial potential functions: Analytical design guidelines. Journal of Intelligent and Robotic Systems, 45(4), 369-394.

Kingston, D., Beard, R., McLain, T., Larsen, M., and Ren, W. (2003). Autonomous vehicle technologies for small fixed wing UAVs. In 2nd AIAA Unmanned Unlimited Systems, Technologies, and Operations. San Diego, California, USA.

Mabrouk, M.H. and McInnes, C. (2008). Nonlinear stability of vortex formation in swarms of interating particles. Phys. rev. 
E, 78(1), 012903.

McInnes, C. (2003). Velocity field path-planning for single and multiple unmanned aerial vehicles. The Aeronautical Journal, 107(1073), 419-426.

McInnes, C. (2007). Vortex formation in swarms of interacting particles. Physical Review E (Statistical, Nonlinear, and Soft Matter Physics), 75(3), 032904.

Ogren, P., Fiorelli, E., and Leonard, N. (2004). Cooperative control of mobile sensor networks: Adaptive gradient climbing in a distributed environment. IEEE Transactions on Automatic Control, 49(8), 1292-1302.

Ollero, A. and Merino, L. (2004). Control and perception techniques for aerial robotics. Annual Reviews in Control, 28(2), 167-178.

Pyragas, K. (1992). Continuous control of chaos by selfcontrolling feedback. Physics Letters A, 170,No. 6, 421-428.

Quigley, M., Goodrich, M., Griffiths, S., Eldredge, A., and Beard, R. (2005). Target acquisition, localization, and surveillance using a fixed-wing mini-UAV and gimbaled camera. In IEEE International Conference on Robotics and Automation, 2600-2605. Barcelona, Spain.

Reif, J. and Wang, H. (1999). Social potential fields: A distributed behavioral control for autonomous robots. Robots and Autonomous Systems, 27(3), 171-194.

Reynolds, C. (1987). Flocks, herds and schools: a distributed behavioural model. Computer Graphics, 21(4), 25-34.

Wallner, O. (2006). Darwin system assessment study. Astrium Summary Report, European Space Agency, 1, 1-18. 